\title{
Study on Virtual Crane Simulation for Monitoring and Prevent Maintenance of Launching and Recovery System
}

\author{
HyunWoo Kim \\ Research Institute of Medium \& Small Shipbuilding \\ 38-6 Noksansandan 232 Kangseo-gu , Busan, South Korea \\ kimhw@rims.re.kr
}

\begin{abstract}
This study is a study to build a virtual crane simulation system for technical research related to remote monitoring and preventive maintenance of marine crane system. In this study, we have studied the construction method of crane system for virtual simulation system. Remote monitoring of the crane system and construction of the actual system for the development of the preventive maintenance technology is necessary, but this system can be an alternative when the crane system can't be built or the system can't be built. The simulation system developed in this study has kinematic and kinematic modelling for CPS based simulation. In order to achieve the near realistic effect of each component according to the operation of the system by developing the closest to the actual crane system. These studies are expected to contribute to the research and application of digital twin systems in the marine sector.
\end{abstract}

Keywords: Simulation, Crane, Virtual, kinematics, Dynamics.

\section{Introduction}

This study is a study to build a virtual crane simulation system for technical research related to remote monitoring and preventive maintenance of marine crane system. The crane system is installed in a ship or offshore structure to launch or recover equipment and cargo. There is a growing demand for marine and offshore plant operations, but due to the maritime nature of the equipment, there may be a problem with the equipment if it fails. Therefore, monitoring and preventive maintenance of the system is very important[1]. Currently, technology development for ICT-based monitoring and maintenance is being done to solve these system problems[2].

In this study, we have studied the construction method of crane system for virtual simulation system. Remote monitoring of the crane system and construction of the actual system for the development of the preventive maintenance technology is necessary, but this system can be an alternative when the crane system can't be built or the system can't be built. The simulation system developed in this study has kinematic and kinematic modelling for CPS based simulation. In order to achieve the near realistic effect of each component according to the operation of the system by developing the closest to the actual crane system[3-4]. In this study, the information of the consumables of the system is not included yet, so the monitoring and preventive maintenance algorithms of the consumables of the system are not applied, but the information about the weight and torque of the system is applied close to the actual through the dynamic analysis of the system.

Chapter 1 introduces the present research and development. Section 2 describes the construction of crane simulation. Section 3 describes the kinematics and dynamics modelling of the crane. Finally, Section 4 concludes the paper and future research plans.

\section{The construction of crane simulation 2.1. Simulation system architecture}

Fig. 1 shows the architecture of a crane simulation system. The crane simulation consists of four modules: Simulation Control Module, Control Algorithm Module, Inverse Kinematics Simulation Module, and Simulation Result and Visualization Module. The simulation control module controls the entire operation of the simulation. It performs various simulation control functions such as start and end of simulation and numerical input. The Control Algorithm module is equipped with control algorithms such as PID and sliding mode to make the simulation cranes move closer to the real world. The Invers Kinematics Simulation module is a module that generates crane motion through kinematics and inverse kinematics analysis so that the crane can have the same motion as the actual one. The Simulation Result and Visualization 
module is a module that shows simulation results so that a person can intuitively check the operation of the crane system. The four modules operate together and communicate the necessary information to the connected modules.

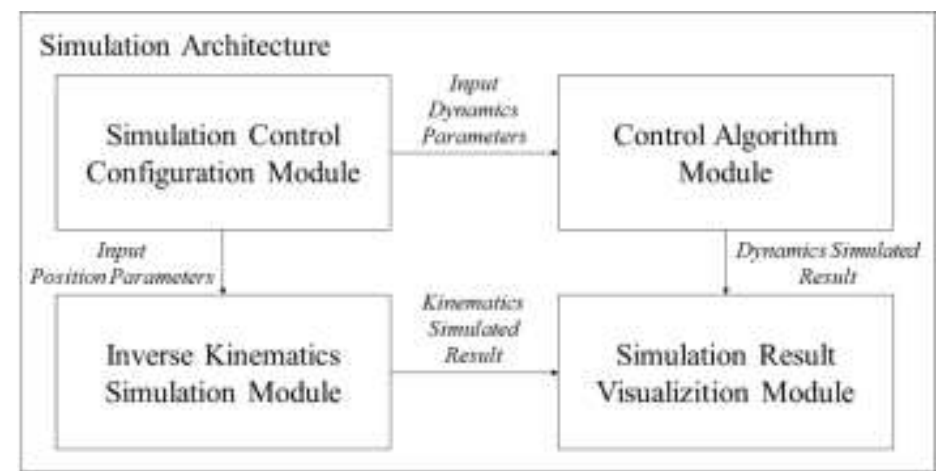

Fig. 1: Simulation system architecture.

\subsection{Simulation program configuration}
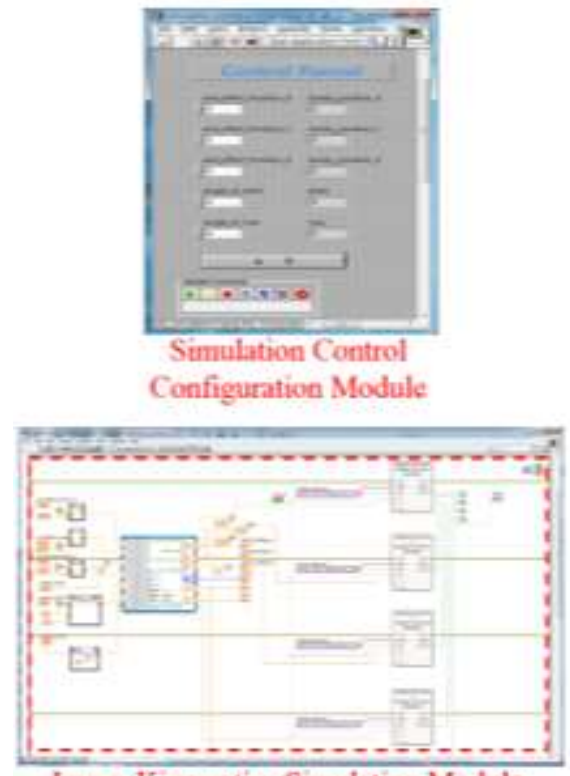

Invers Kinematics Simulation Moduls

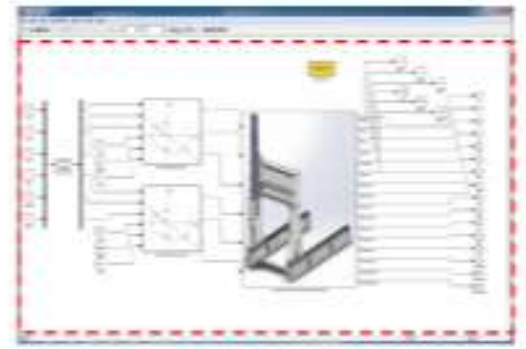

Control Algorithm Module

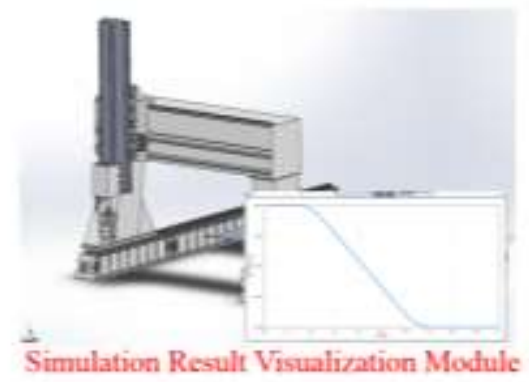

Fig. 2: Simulation program configuration.

A simulation program was developed according to the design of the aforementioned simulation system architecture. The developed program is shown in Fig. 2. The control input of each joint automatically generated generates the input torque through the Control Algorithm module and generates the position and attitude information of each joint through the Invers Kinematics Simulation module. The simulation result through the control algorithm module and the inverse kinematics simulation module represents the current state through the simulation result and visualization module. The simulation control module connects simulations of each module and exchanges data with the LabVIEW program. Simulation control is also performed through the interface with the user. The control algorithm module is a module for control of the most important crane system. It calculates torque of actuator according to position, velocity and acceleration of each axis and transmits it to visualization simulation. The control algorithm module is in Simulink form of MATLAB and is configured to simulate the data exchange by integrating with LabVIEW control module. The inverse kinematic model changes the end-effect of the system to the movement position of each joint and transmits it to the simulation visualization module. The simulation visualization module links LabVIEW and Solidworks and connects the simulated result to 3D modelling, so that the user can check the movement. 


\section{Mathematical modelling}

\subsection{Kinematics}

The kinematic model of the crane is shown in Eq. (1).

$$
A_{0}^{7}=\left[\begin{array}{cccc}
-c \theta_{5}^{*} s \theta_{7}^{*}-c \theta_{6}^{*} c \theta_{7}^{*} s \theta_{5}^{*} & -c \theta_{5}^{*} s \theta_{7}^{*}+c \theta_{6}^{*} s \theta_{5}^{*} s \theta_{7}^{*} & -s \theta_{5}^{*} s \theta_{6}^{*} & d_{3}^{*}-d_{7} s \theta_{5}^{*} s \theta_{6}^{*} \\
s \theta_{5}^{*} s \theta_{7}^{*}-c \theta_{5}^{*} c \theta_{6}^{*} c \theta_{7}^{*} & s \theta_{5}^{*} c \theta_{7}^{*}-c \theta_{5}^{*} c \theta_{6}^{*} s \theta_{7}^{*} & -c \theta_{5}^{*} s \theta_{6}^{*} & d_{2}^{*}-d_{7} c \theta_{5}^{*} s \theta_{6}^{*} \\
-c \theta_{7}^{*} s \theta_{6}^{*} & s \theta_{6}^{*} s \theta_{7}^{*} & -c \theta_{6}^{*} & d_{1}-d_{4}^{*}-d_{7} c \theta_{6}^{*} \\
0 & 0 & 0 & 1
\end{array}\right]=\left[\begin{array}{cccc}
r_{11} & r_{12} & r_{13} & p_{x} \\
r_{21} & r_{22} & r_{23} & p_{y} \\
r_{31} & r_{32} & r_{33} & p_{z} \\
0 & 0 & 0 & 1
\end{array}\right]
$$

The conversion matrix of Eq. (1) can be obtained by the combination of the translation matrix and the rotation matrix of a 6-axis crane using the Denavit-Hartenberg notation. The crane is composed of six axes. The three axes are composed of orthogonal coordinate axes that move linearly in the $\mathrm{x}, \mathrm{y}$, and $\mathrm{z}$ axes. The remaining three axes are composed of rotation axes that rotate pitch, roll, and yaw. In the equation (1), a variable with a star is a variable that changes according to the movement of the actuator. The inverse kinematic solution of this system uses a method of isolating the joint variables to be obtained by maximizing the function form of the matrix components. This method has an advantage that the joint variable value can be easily obtained by using the geometric characteristics of the system.

$$
\begin{gathered}
\theta_{5}^{*}=\tan ^{-1}\left(\frac{r_{13}}{r_{23}}\right) \text { if } \sin \theta_{6}^{*}>0 \text { or } \theta_{5}^{*}=\tan ^{-1}\left(\frac{-r_{13}}{-r_{23}}\right) \text { if } \sin \theta_{6}^{*}<0 \\
\theta_{6}^{*}=\tan ^{-1}\left( \pm \frac{\sqrt{1-r_{33}^{2}}}{r_{33}}\right) \\
\theta_{7}^{*}=\tan ^{-1}\left(\frac{r_{32}}{r_{31}}\right) \text { if } \sin \theta_{6}^{*}>0 \text { or } \theta_{7}^{*}=\tan ^{-1}\left(\frac{-r_{32}}{-r_{31}}\right) \text { if } \sin \theta_{6}^{*}<0 \\
d_{2}^{*}=p_{y}+d_{7} c \theta_{5}^{*} s \theta_{6}^{*} \\
d_{3}^{*}=p_{x}+d_{7} s \theta_{5}^{*} s \theta_{6}^{*} \\
d_{4}^{*}=p_{z}+d_{7} c \theta_{5}^{*} s \theta_{6}^{*}-d_{1}
\end{gathered}
$$

\subsection{Dynamics}

The equation of motion can be expressed as a Lagrangian equation of motion such as Eq. (3).

$$
M(q) \ddot{q}+C(q, \ddot{q})+G(q)+F_{f}+D=u
$$

In Eq. (3), the coefficients of each term are composed of $M(q) \in R^{6 \times 6}, C(q, \ddot{q}) \in R^{6 \times 1}, G(q) \in R^{6 \times 1}, F_{f} \in R^{6 \times 1}, D \in$ $R^{6 \times 1}, u \in R^{6 \times 1}$ type matrix, and the matrix of each term is summarized as follows. $M(q)$ is a positive symmetric matrix that defines the inertial matrix, and $C(q, \ddot{q})$ is a matrix of vectors of Coriolis force and centripetal force. $G(q)$ is a vector matrix representing gravity, $F_{f}$ is a matrix representing friction, and $D$ is a matrix representing a disturbance. Finally, $u$ is a matrix of input and torque.

$$
M(q)=\left[\begin{array}{cccccc}
m_{t} & 0 & 0 & 0 & m_{t} l c \theta & 0 \\
0 & m_{y} & 0 & m_{y} l c \emptyset & 0 & 0 \\
0 & 0 & m_{z} & m_{z} l s \emptyset & m_{z} l s \theta & 0 \\
0 & m_{y} l c \emptyset & m_{z} l s \emptyset & m_{6} l^{2}+J_{x}+m_{y} l^{2} c^{2} \emptyset+m_{z} l^{2} s^{2} \emptyset & m_{z} l s \emptyset s \theta & 0 \\
m_{t} l c \theta & 0 & m_{z} l s \theta & m_{z} l^{2} s \emptyset s \theta & m_{6} l^{2}+J_{y} m_{t} l^{2} c^{2} \theta+m_{z} l^{2} s^{2} \emptyset & 0 \\
0 & 0 & 0 & 0 & 0 & m_{6} R^{2}+J_{z}
\end{array}\right],
$$




$$
\begin{aligned}
& C(q, \ddot{q})=\left[\begin{array}{c}
-m_{t} l \dot{\theta}^{2} s \theta \\
-m_{y} l \dot{\phi}^{2} s \emptyset \\
m_{z} l \dot{\theta}^{2} c \theta+-m_{z} l \dot{\emptyset}^{2} c \emptyset \\
-m_{y} l \dot{y} \dot{\phi} s \emptyset+m_{z} l \dot{z} \dot{\emptyset} c \emptyset-2 m_{y} l^{2} \dot{\phi}^{2} c \emptyset s \emptyset+m_{z} l^{2} \dot{\theta}^{2} c \theta s \emptyset+m_{z} l^{2} \dot{\theta} \dot{\varnothing} s \theta c \emptyset+2 m_{z} l^{2} \dot{\emptyset}^{2} c \emptyset \\
-m_{t} l \dot{x} \dot{\theta} s \theta-2 m_{t} l^{2} \dot{\theta}^{2} c \theta s \theta+m_{z} l^{2} \dot{\theta}^{2} s \theta s \theta+m_{z} l^{2} \dot{\emptyset}^{2} c \emptyset s \theta+m_{z} l^{2} \dot{\theta} \dot{\emptyset} s \emptyset c \theta \\
0
\end{array}\right], \\
& G(q)=\left[\begin{array}{c}
0 \\
0 \\
m_{z} g \\
m_{6} g l s \emptyset \\
m_{6} g l s \theta \\
0
\end{array}\right], \quad F_{f}=\left[\begin{array}{c}
F_{f x} \\
F_{f y} \\
F_{f z} \\
\tau_{f \varnothing} \\
\tau_{f \theta} \\
\tau_{f \varphi}
\end{array}\right], \quad D=\left[\begin{array}{c}
d_{x} \\
d_{y} \\
d_{z} \\
d_{\varnothing} \\
d_{\theta} \\
d_{\varphi}
\end{array}\right], \quad u=\left[\begin{array}{c}
F_{x} \\
F_{y} \\
F_{z} \\
\tau_{\varnothing} \\
\tau_{\theta} \\
\tau_{\varphi}
\end{array}\right]
\end{aligned}
$$

\section{Conclusion}

In this study, a dynamic model of the system was established to implement the CPS - based crane simulation. And kinematic analysis was applied to confirm the condition of the motion of the system. This allows us to confirm the movement speed of the system's motion and movement range cranes. In the next study, we plan to apply the same controller as the actual controller of the system, and implement a digital twin type system. In addition, the system will be linked through the remote monitoring data of the simulation system and the actual system, and the alarm system for preventive maintenance will be developed. These studies are expected to contribute to the research and application of digital twin systems in the marine sector.

\section{Acknowledgements}

This research was supported by the Ministry of Trade, Industry \& Energy(MOTIE), Korea Institute for Advancement of Technology(KIAT) through the Encouragement Program for The Industries of Economic Cooperation Region. (P0002161)

\section{References}

[1] Yanming Li and Chengliang Liu, "Integrating field data and 3D simulation for tower crane activity monitoring and alarming," Automation in Construction, vol. 27, pp.111-119, 2012..

[2] Y. N. Burali, "PLC based industrial crane automation \& monitoring," International Journal of Engineering and Science, vol. 1, no. 3, pp. 01-04, 2012..

[3] Michael Schluse and Juergen Rossmann, "From simulation to experimentable digital twins: Simulation-based development and operation of complex technical systems," in 2016 IEEE International Symposium on Systems Engineering (ISSE), IEEE, 2016,

[4] Jorge Posada, Carlos Toro, Inigo Barandiaran, David Oyarzun, Didier Stricker, Raffaele de Amicis, Eduardo B. Pinto, Peter Eisert, Jurgen Dollner, Ivan Vallarino, "Visual computing as a key enabling technology for industrie 4.0 and industrial internet," IEEE computer graphics and applications, vol. 35, no. 2, pp. 26-40, 2015. 


\section{Appendix}

Variable setting and definition of crane system
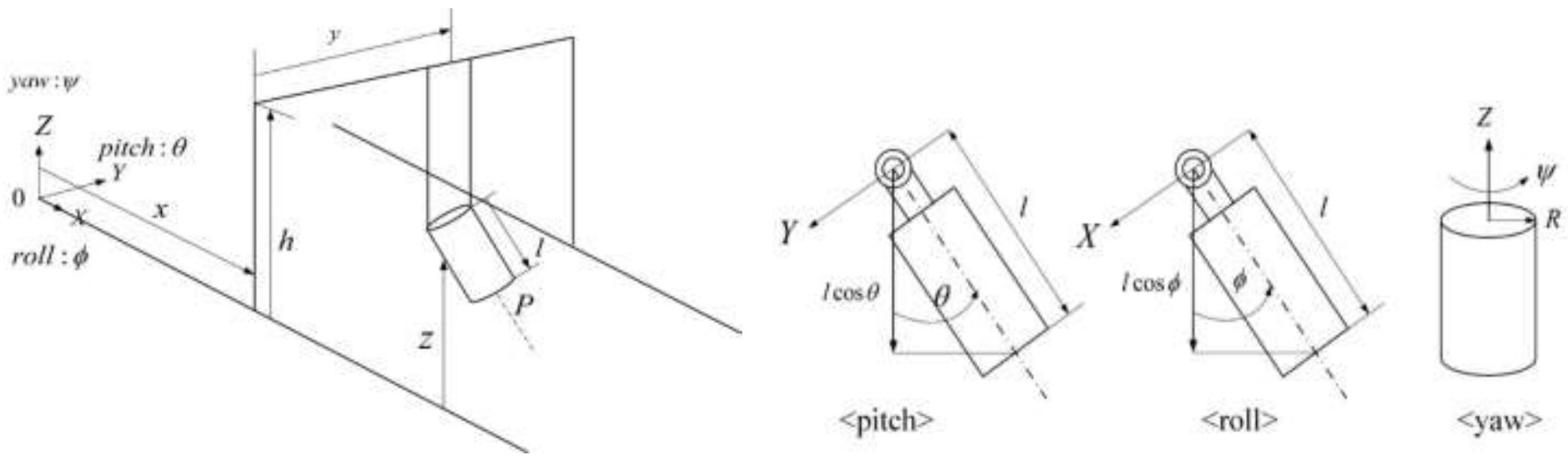

Fig. 3: Variable setting of crane system.

Define variables for each element for dynamic modelling:

1) $m_{1}:$ mass of the left column frame

2) $m_{2}:$ mass of the right column frame

3) $m_{3}:$ mass of the upper frame

4) $m_{4}:$ mass of the fixed part of upper head frame

5) $m_{5}:$ mass of the moving part of upper head frame

6) $m_{6}:$ mass of the head frame

7) $m_{t}=m_{1}+m_{2}+m_{3}+m_{4}+m_{5}:$ mass of the total frame

8) $m_{y}=m_{4}+m_{5}+m_{6}:$ mass of the upper head and head frame

9) $m_{z}=m_{5}+m_{6}:$ mass of the upper head and head frame

10) $J_{x}=J_{y}=\frac{1}{3} m_{6} l^{2}:$ moment of inertia of the head part with respect to $\mathrm{x}$ and $\mathrm{y}$ axes

11) $J_{x}=\frac{1}{2} m_{6} R^{2}:$ moment of inertia of the head part with respect to $\mathrm{z}$ axis

12) $h:$ height of the frame

13) $l:$ length of the head part

14) $R:$ radius of the head part 